\title{
Failure Mechanisms of Nanoparticle Reinforced Metal Matrix Composite
}

\author{
A. K. Basak ${ }^{1, a}$, A. Pramanik ${ }^{2, b}$, and M. N. Islam ${ }^{2, c}$ \\ ${ }^{1}$ Adelaide Microscopy, University of Adelaide, Adelaide, SA, Australia \\ ${ }^{2}$ Department of Mechanical Engineering, Curtin University, Bentley, WA, Australia

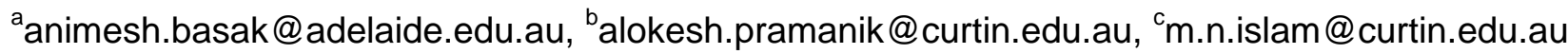

Keywords: nanoparticle, metal matrix composite, fracture

\begin{abstract}
The quest for the advanced functional material of superior functionality for advanced structure is being driven in various fronts of engineering materials. One of such front is metal matrix composite (MMC) which has already been proven as one of the most productive field in that respect. With the advance of technology, now it is possible to reinforce the MMCs with nano-sized particles compared to conventional micron-sized ones. However, the addition of nanoparticle in the MMC to improve its mechanical properties is not unconditional. To achieve positive gain by adding nanoparticles in the MMCs, all the influencing factors should be taken into consideration. The present paper reviews the failure mechanisms of nanoparticles reinforced MMCs in light of its strengthening mechanisms.
\end{abstract}

\section{Introductions}

Recent developments in nanostructured material production of various metals and ceramics have enhanced research activities to create multi-functional engineering materials as ‘bottom-up' approach e.g., by designing structures at nano-scale. This also motivates the development of nanoparticle reinforced MMCs-one of the rapidly evolving areas of composites research [1-3]. In that context, it is important to understand the nanostructured materials and their interaction with the matrix. In the MMCs, second phase particles are added to enhance elastic modulus and yield strength of the matrix. In contracts with micron-particle reinforced MMCs, nanoparticle reinforced MMCs are found to be more unpredictable, sometimes diminishing the properties [4-7]. To get the positive benefit of nanoparticle addition in MMCs, the first challenge is to retain the nano-size of the particles in MMCs after processing. Traditionally, micron-sized reinforced MMCs have been produced mainly via powder metallurgy route or molten metal processing [7-9]. With powder metallurgy processing, the matrix alloy powder is blended with reinforcement particles to achieve homogeneous mixture and subjected to high temperature isostatic pressing. With nanoparticle addition, isostatic pressing became more difficult as the flow stress decrease drastically as well as particle agglomeration/coalition became a major issue [10]. Molten metal processing route also suffer from the same problem as finer particles have a higher tendency to segregate into clusters. However, it is possible to disperse the nanoparticles uniformly by forming the reinforcement particle in-situ in the metal matrix as well as other techniques like stirring, ultrasonic vibration methods etc. [11-15].

Challenges in nanoparticle reinforced MMCs. The critical issue in nanoparticle reinforced MMCs is the retention of nano-size in the composite after processing. There are number of factors controlling this issue as discussed hereafter:

Critical size of nanoparticles. It is not like that, the fine the particles, the greater the benefits. There is 'critical size' below which the addition of particles is injurious as explained as negative 'Hall-Petch' effect. Therefore to get the optimum benefit, nanoparticles around critical size range should be considered [16].

Agglomeration of nanoparticles. As the particle became finer, e.g., in the range of nano-scale, the surface energy of the particles increase by couple of orders of magnitude compared to micron-sized oncers. These nanoparticles are highly reactive with its surrounding environments and 
introduce strong instability in the system. The wettability of the nanoparticles by molten metal matrix is also different compared to micron-sized ones [17-19]. Though dislocation mean free path length and average distance between dislocations obstacles reduce with the increase of nanoparticle content, however, this effect became neutralized by particle coalescence and segregation [13]. It seems that, a random particle distribution exhibits the best combination of dislocation mean free path length and average obstacle distance.

Interface de-bonding. One of the most important issues in nanoparticle reinforced MMCs is the bonding between the matrix and the particle, as this interface is the weakest link in the whole structure. This is essential for an effective load transfer from matrix- to- particle and delaying the onset of particle-matrix de-bonding. Both of these have a profound effect on the strength and stiffness of the composite. As the nanoparticles are chemically reactive due to this enhanced surface energy, they are very prone to oxidation [20-22]. Once oxides, it is difficult to get enough wettability by the molten metal matrix and cause enhanced floating instead of immersion in the matrix. Two types of nanoparticles are being widely used in the MMCs: (1) metallic such as $\mathrm{Cu}, \mathrm{Ag}$, Ni etc. and (2) ceramic such as $\mathrm{TiO}_{2}, \mathrm{Al}_{2} \mathrm{O}_{3}, \mathrm{SiC}$, TiB etc. Generally, pure metallic particles have a common feature that they bond to metal matrix well due to ease of diffusion $[18,23]$. In the case of ceramic nanoparticle reinforced MMCs the bonding between particles and matrix is rather weak. Such inert reinforcements are lack of any metallurgical bond to the matrix. This can be overcome by careful processing parameters and avoiding oxidation of the nanoparticles.

\section{Effect of nanoparticles on physical and mechanical properties of MMC}

Microstructure. Nanoparticle addition in the MMCs is expected to offer grain refinement due to pinning effect in the grain boundaries. The grain refinement and strong multidirectional thermal stress at the particle-matrix interface are important factors which play a significant role in the high strength of the composites. For example, SiC particles have grain-refined strengthening effect, which is improved with increasing volume fraction since they act as the heterogeneous nucleation catalyst for aluminium [6-12]. However, there are reports which show the inability of nanoparticles to serve as either nucleation sites or obstacles to grain growth during solid state cooling and no significant change in grain size compared to the corresponding monolithic alloy [17, 24].

Hardness. Nanoparticle reinforced MMC usually exhibit higher microhardness than that of the unreinforced matrix alloy. The increase in hardness can be ascribed as follows: (1) strengthening mechanisms arising from restricting or impeding the motion of dislocation, (2) presence and near-uniform distribution of second-phase particles achieved by optimum processing, and (3) a refinement in grain size.

Strength. The yield and ultimate strength of MMCs increase with the addition of nanoparticles as smaller particles tend to be stronger. This in turn diminishes the extent of particle fracture during composite deformation and increases the hardening rate in tension. For a given geometry of plastic flow, finer microstructures lead to greater strain gradients in the matrix during deformation due to differential matrix/reinforcement thermal contraction from processing temperatures.

\section{Failure mechanisms}

It is well-known that mechanical properties of the particle reinforced MMCs are strongly affected by local events such as voids nucleation at the reinforcement/matrix interface, fracture of the reinforcement, initiation and propagation of matrix shear slip bands and the linkage of these local damage and interfacial de-cohesion [9]. The efficiency of the stress transfer from the matrix to particles plays an important role in determining the mechanical performance. Various nano-scale deformation modes including slip bands, surface roughening, and cracking of nanoparticles are observed during tensile loading. The amount of slip bands and the average surface roughness were found to increase with increasing applied strain due to plastic deformation in the matrix through the 
formation of matrix slip bands. The matrix slip bands create sharp discontinuities on the order of 5-15 $\mathrm{nm}$ at the surface of particle that may cause the stress concentration, cracking and distortion of the particles. The multiple fractures in the nanoparticles are synergistic effect of both the stress transfer mechanism and the stress concentration mechanism due to the presence of slip bands [9-11].

For nanoparticle reinforced MMCs, de-cohesion is the primary mode of damage mechanisms. Most of the structural material fails by a process known as micro-void coalescence. The micro-voids nucleates at region of localized strain discontinuity like second phase particles, inclusion and grain boundary dislocation piles up. As the strain in the material increases micro-voids grow, coalescence and form a continuous fracture surface. This is evident by a closer look of the facture surface with electron microscopy that matrix material in the void undergoes extensive plastic deformation by shear and is drawn out to form a sharp shear lip Void coalescence occurred by the progressive growth and impingement of micro-voids and the fracture surface shows dimples of different diameter. The largest dimple forms around the reinforced particle. The size of the dimples on a fracture surface depends on the number and distribution of micro-voids that are nucleated. When the nucleation sites are less (reinforcement vol. \% is less) and widely spaced the micro-void grow to a larger size. Small dimples are formed when numerous nucleating sites are activated and the micro-voids join before they have an opportunity to grow to a larger size [17]. Fracture under uni-axial tensile load results in equiaxed dimples. Oval-shaped dimples are observed on the wall of large elongated dimples. Oval dimple is formed when a small surface void intersect the wall of a larger void.

Regarding the trend in tensile failure strain, it has been shown that the nanoparticles provide sites where cleavage cracks are opened ahead of the advancing crack front. This cleavage crack opening dissipates the stress concentration that would otherwise exist at the crack front and alters the local effective stress state from plane strain to plane stress in the neighbourhood of the crack tip [17, 14]. The trend in tensile failure strain can also be attributed to the partial reactivity between nanoparticles and metal matrix. Regarding the trend in compressive failure strain, compressive shear buckling of nanoparticles aided in dispersing localized stored energy during compressive deformation. This allowed nanoparticle reinforced MMCs to globally absorb relatively large amounts of strain energy during compressive deformation [12-13]. At clustered region, the amount of reinforcement volume fraction is larger which imposed a higher plastic constraint to the plastic deformation of the ductile matrix. Due to this large hydrostatic stress developed in clustered regions, which increases the maximum principal stress in the particle leading to the early damage of the composite by reinforcement particle cracking. The necessary condition for interface de-cohesion is that the local elastic strain energy released during de-cohesion should be sufficient to create the new free surface at the interface. The above condition holds good for particle of few length of nm or smaller $[12,18]$. For most of the materials, room temperature damage is prevalent by reinforcement fracture but the damage mechanism changes to void nucleation by interface decohesion at a temperature around $200^{\circ} \mathrm{C}$ and above. [16]. At high temperatures $\left(300^{\circ} \mathrm{C}\right)$ damage occurs by matrix voiding in the vicinity of reinforced particle and the interface de-cohesion. At high operating temperature reinforcement particle played a minor role in the failure process, as the plastic strain being accumulated by the extremely ductile matrix.

\section{Future aspects}

High volume and high rate fabrication of nanoparticles and better understanding of their fundamental behaviours make them viable as a large throughput MMC fabrication. Besides that, costs of nanoparticle reinforced MMCs are also coming down with technological advances. It is anticipated that as applications for nanoparticles and their composites increase the cost will be dramatically reduced. The know-how in the fabrication of traditional fibre composites have clearly demonstrated that the development of a science base for manufacturing is indispensable and more fundamental investigation on that is foreseen. 


\section{Concluding remarks}

This present paper has reviewed the possible failure mechanisms on nanoparticle reinforced metal matrix composite. The main challenge in nanoparticle reinforced MMCs is to retain the nano-size of the reinforced particles in the metal matrix after process with our any agglomeration. In general nanoparticle reinforced MMC offer (1) improvement in strength, (2) improvement of fracture toughness, (3) improvement of creep resistance, thermal shock resistance, and wear resistance, and (4) enhance dimensional stability at high temperatures. The main fracture mechanism of nanoparticle reinforced MMC under different loading condition is trans-granular fracture compared to inter-granular fracture of monolithic one. Particle-matrix debonding is the primary mode of material deformation at high temperature whereas particle cracking at low temperature.

\section{References}

[1] M. Paramsothy, J. Chan, R. Kwok, M. Gupta, Nanomater. 2 (2012) p. 147

[2] L.C. Zhang, E.C.S. Kiat, A. Pramanik, Adv.Mater. Res, 76 (2009) p. 212

[3] A. M. Dongare, B. LaMattina, Adv. Nanocompo. Technol. 2(2009) p. 227

[4] B. S. kumar, L. Ramesh, Int. J. Eng. Res. Technol. 6 (2012) p. 254

[5] Y. Tanaka, J.-M. Yang, Y.F. Liu, Y. Kagawaa, Scripta Mater. 56 (2007) p. 209

[6] H.R. Hafizpour, M. Khoeini, J. American Sci. 7 (7) (2011) p. 547

[7] A. Pramanik, A. Basak, Adv. Mater. Res. 651(2013) p.334

[8] A. Pramanik, L.C. Zhang, J.A. Arsecularatne, Key Eng. Mater. 340 (2007) p.563.

[9] E. T. Thostenson, C. Li, T.-W. Chou, Comp. Sci. Technol. 65 (2005) p. 491

[10] J. R. Greer, J. Th.M. De Hosson, Pro. Mat. Sci. 56(6) (2011) p. 654

[11] G. Cao, X. Chen, J. W. Kysar, D. Lee, Y. X. Gan, Mech. Res. Comm. 34 (2007) p. 275

[12] A. Pramanik, L.C. Zhang, J.A. Arsecularatne, Comp. Sci. and Tech. 68(6) (2008) p. 1304

[13] A. Pramanik, A. Basak, Adv. Mater. Res. 651(2013) p.350

[14] C.-F. Chen, P.-W. Kao, L. Chang, N.-J. Ho, Mater. Trans. 51 (5) (2010) p. 933

[15] A. Mortensen, J. Llorca, Annu. Rev. Mater. Res. 40 (2010) p. 43

[16] G.E. Dieter, Mechanical Metallurgy (1961) McGraw-Hill, NewYork

[17] E. T. Thostenson, C. Li, T.-W. Chou, Compo. Sci. Technol. 65 (2005) p. 491

[18] D. Bozic, J. Stasic, B. Dimcic, M. Vilotijevic, V. Rajkovic, Bull. Mater. Sci. 34 (2) (2011) p. 217

[19] J. Babu Rao, Dil Kush, NRMR Bhargava, J. Miner. Mater. Character. Eng. 11 (5) (2012) p. 529

[20] J. Yang, Z. Zhang, K. Friedrich, A. K. Schlar, App. Phy. Lett. 91(2007) 011901.

[21] C. Fan, L. Kecskes, T. Jiao, H. Choo, A. Inoue, P. Liaw, Mater. Trans. 47 (3) (2006) p. 817

[22] A. Pramanik, M.N. Islam, A. Basak, G. Littlefair, Adv. Mater. Res. 651(2013) p.338

[23] S. W. Lee, H. J. Choi, Y. Kim, D.H. Bae, Mat. Sci. Eng. A 449-451 (2007) p. 782

[24] J.-l. Tsai, H. Hsiao, Y.-l. Cheng, J. Comp. Mater. 44 (4) (2010) p.625 